\title{
HYBRID FIXED POINT THEORY AND EXISTENCE OF EXTREMAL SOLUTIONS FOR PERTURBED NEUTRAL FUNCTIONAL DIFFERENTIAL EQUATIONS
}

\author{
Bapurao C. Dhage
}

\begin{abstract}
In this paper, some hybrid fixed point theorems are proved which are further applied to first and second order neutral functional differential equations for proving the existence results for the extremal solutions under the mixed Lipschitz, compactness and monotonic conditions.
\end{abstract}

\section{Statement of the problems}

The functional differential equations (in short FDE) is a topic of great interest since long time in the theory of differential equations. These equations are modeled on a dynamical system in which the present state is determined by the past state of the related dynamical systems. During the last half century a significant efforts have been applied to study functional differential equations, i.e., equations containing derivatives of the solutions and dependencies on the solutions having non-local character (the right hand side depend not only on the solutions, but also on the "prehistory" as well). Indeed, such models based on the boundary value problems for equations with deviating arguments or integro-differential equations provide the most adequate and accurate description of different processes in physics, economics, bio-mathematics and social sciences. Therefore their study is of great importance and applications.

It is well known that when there are deviations of the differentiated functions from the solutions (which characterizes neutrality), the questions of the existence of solutions are essentially more complex for the study when in the case of FDE with deviating argument only in the right hand sides. Such results for NFDE are of definite theoretical and practical importance. The exhaustive treatment of NFDEs appear in the monographs like Hale [7], Henderson [9] and a recent survey of Ntouyas [11]. The nonlinear NFDEs are generally studied for

Received August 22, 2006.

2000 Mathematics Subject Classification. 47H10, 34A60.

Key words and phrases. Neutral functional differential equation, extremal solutions. 
the existence results under the Lipschitz or Carathéodory or monotonic or the sum of two Lipschitz or Carathéodory conditions of nonlinearities. Sometimes it may happen that the nonlinearity involved in a NFDE is neither Lipschitz, Carathéodory nor monotonic and can not be decomposed as a sum of a Lipschitz and a Carathéodory nonlinearity as well. Then in such cases, it seems to be impossible to prove the existence results using the existing fixed point theorems from nonlinear functional analysis. This is the main motivation of the present paper and here in this paper, we prove a hybrid fixed point theorem which covers a situation mentioned above and apply it to prove the existence theorems for extremal solutions of certain neutral functional differential equations.

Let $\mathbb{R}$ be the real line and let $I_{0}=[-\delta, 0], \delta>0$, and $I=[0, T]$ be two closed and bounded intervals in $\mathbb{R}$. Denote $J=I_{0} \cup I=[-\delta, T]$. Let $\mathcal{C}$ denote the Banach space of continuous real-valued functions on $I_{0}$ with the supremum norm $\|\cdot\|_{\mathcal{C}}$ defined by

$$
\|x\|_{\mathcal{C}}=\sup _{t \in I_{0}}|x(t)|
$$

For any continuous real-valued function $x$ on $J$ and for any $t \in I$, let $x_{t}: I_{0} \rightarrow \mathbb{R}$ be a function defined by

$$
x_{t}(\theta)=x(t+\theta), \quad-\delta \leq \theta \leq 0 .
$$

Given a function $\phi \in \mathcal{C}$, in section 2, we consider the following first order neutral functional differential equation,

$$
\left.\begin{array}{rl}
\frac{d}{d t}\left[x(t)-f\left(t, x_{t}\right)\right] & =g\left(t, x_{t}\right)+k\left(t, x_{t}\right) \text { a.e. } t \in I \\
x_{0} & =\phi,
\end{array}\right\}
$$

where $f, g, k: I \times \mathcal{C} \rightarrow \mathbb{R}$.

Later, we study the following second order functional neutral differential equation,

$$
\left.\begin{array}{rl}
\frac{d}{d t}\left[x^{\prime}(t)-f\left(t, x_{t}\right)\right] & =g\left(t, x_{t}\right)+k\left(t, x_{t}\right) \text { a.e. } t \in I \\
x_{0} & =\phi, \quad x^{\prime}(0)=\eta,
\end{array}\right\}
$$

where $f, g, k: I \times \mathcal{C} \rightarrow \mathbb{R}$.

The NFDEs (1.1) and (1.2) have already been studied in Sficas et al. [13] and Ntouyas et al. [14] for the existence results. There, all the nonlinearities involved in the equations are required to be continuous on the respective domains of their definition. The NFDEs (1.1) and (1.2) further include the NFDEs

$$
\left.\begin{array}{rl}
\frac{d}{d t}\left[x(t)-f\left(t, x_{t}\right)\right] & =g\left(t, x_{t}\right) \text { a.e. } t \in I \\
x_{0} & =\phi
\end{array}\right\}
$$


and

$$
\left.\begin{array}{c}
\frac{d}{d t}\left[x^{\prime}(t)-f\left(t, x_{t}\right)\right]=g\left(t, x_{t}\right) \text { a.e. } \quad t \in I \\
x_{0}=\phi, \quad x^{\prime}(0)=\eta,
\end{array}\right\}
$$

as the special cases. The NFDEs (1.3) and (1.4) have also been studied in Ntouyas et al. [12] and Sficas and Ntouyas [13] for the existence results under uniform continuity and Carathéodory conditions of the nonlinearities $f$ and $g$ respectively. In this paper, we prove the existence results for extremal solutions for the NFDEs (1.1) and (1.2) under the mixed Lipschitz, Carathéodory and monotonic conditions of the nonlinearities involved in them. We do not require the continuity of all functions in the NFDEs (1.1) and (1.2). Our results includes the existence results for extremal solutions of the NFDEs (1.3) and (1.4) under the weaker conditions which are again new to the literature.

\section{Preliminaries}

Let $X$ be a Banach space with the norm $\|\cdot\|$. A mapping $A: X \rightarrow X$ is called $\mathcal{D}$-Lipschitz if there exists a continuous nondecreasing function $\psi: \mathbb{R}^{+} \rightarrow \mathbb{R}^{+}$ satisfying

$$
\|A x-A y\| \leq \psi(\|x-y\|)
$$

for all $x, y \in X$ with $\psi(0)=0$. In the special case, when $\psi(r)=q r(q>0), A$ is called a Lipschitz with the Lipschitz constant $q$. In particular, if $q<1$, then $A$ is called a contraction with the contraction constant $q$. Further, if $\psi(r)<r$ for all $r>0$, then $A$ is called a nonlinear $\mathcal{D}$-contraction on $X$. For convenience, we call the function $\psi$ to be a $\mathcal{D}$-function of $A$ on $X$.

An operator $T: X \rightarrow X$ is called compact if $\overline{T(X)}$ is a compact subset of $X$. Similarly, $T: X \rightarrow X$ is called totally bounded if $T$ maps the bounded subsets of $X$ into the relatively compact subsets of $X$. Finally, $T: X \rightarrow X$ is called completely continuous operator, if it is continuous and totally bounded operator on $X$. It is clear that every compact operator is totally bounded, but the converse may not be true. However, the two notions are equivalent on the bounded subsets of $X$.

The Kuratowskii measure of noncompactness $\alpha$ of a bounded set $S$ in $X$ is a nonnegative real number $\alpha(S)$ defined by

$$
\alpha(S)=\inf \left\{r>0: S=\bigcup_{i=1}^{n} S_{i}, \text { and } \operatorname{diam}\left(S_{i}\right) \leq r, \forall i\right\} .
$$

The details of measures of noncompactness and their properties appear in Granas and Dugundji [5], Deimling [4] and Zeidler [15].

Definition 2.1. A mapping $T: X \rightarrow X$ is called condensing (resp. countably condensing), if for any bounded (resp. bounded and countable) subset $S$ of $X$, $T(S)$ is bounded and $\alpha(T(S))<\alpha(S)$, if $\alpha(S)>0$. 
Note that contraction and completely continuous mappings are condensing, but the converse may not be true. Again, every condensing mapping is countably condensing but the converse may not be true.

A non-empty closed set $K$ in a Banach space $X$ is called a cone if (i) $K+K \subseteq$ $K$, (ii) $\lambda K \subseteq K$ for $\lambda \in \mathbb{R}, \lambda \geq 0$ and (iii) $\{-K\} \cap K=\{0\}$, where 0 is the zero element of $X$. We introduce an order relation $\leq$ in $X$ as follows. Let $x, y \in X$. Then $x \leq y$ if and only if $y-x \in K$. A cone $K$ is called normal if the norm $\|\cdot\|$ is semi-monotone increasing on $K$, that is, there is a constant $N>0$ such that $\|x\| \leq N\|y\|$ for all $x, y \in K$ with $x \leq y$. It is known that if the cone $K$ is normal in $X$, then every order-bounded set in $X$ is norm-bounded. Similarly, the cone $K$ in $X$ is called regular if every monotone increasing (resp. decreasing) order bounded sequence in $X$ converges in norm. The details of cones and their properties appear in Heikkilä and Lakshmikantham [8].

For any $a, b \in X, a \leq b$, the order interval $[a, b]$ is a set in $X$ given by

$$
\{a, b]=\{x \in X: a \leq x \leq b\} .
$$

Definition 2.2. Let $X$ and $Y$ be two ordered Banach spaces. A mapping $T: X \rightarrow Y$ is said to be nondecreasing or monotone increasing if $x \leq y$ implies $T x \leq T y$ for all $x, y \in[a, b]$.

We use the following two fixed point theorems in the sequel.

Theorem 2.1. Let $[a, b]$ be a norm-bounded order interval in an ordered Banach space $X$ and let $T:[a, b] \rightarrow[a, b]$ be a continuous and countably condensing mapping. If $T$ is nondecreasing, then $T$ has the least fixed point $x_{*}$ and the greatest fixed point $x^{*}$ in $[a, b]$ and the sequences $\left\{T^{n}(a)\right\}$ and $\left\{T^{n}(b)\right\}$ converge to $x_{*}$ and $x^{*}$ respectively.

Proof. The proof can be obtained by using essentially the same arguments that given in Dhage [1] with appropriate modifications. We omit the details.

Theorem 2.2. (Heikkilä and Lakshmikantham [8]) Let $[a, b]$ be an order interval in a subset $Y$ of an ordered Banach space $X$ and let $Q:[a, b] \rightarrow[a, b]$ be a nondecreasing mapping. If each sequence $\left\{Q x_{n}\right\} \subseteq Q([a, b])$ converges in $Y$, whenever $\left\{x_{n}\right\}$ is a monotone sequence in $[a, b]$, then the sequence of $Q$ iteration of a converges to the least fixed point $x_{*}$ of $Q$ and the sequence of $Q$-iteration of $b$ converges to the greatest fixed point $x^{*}$ of $Q$. Moreover,

$$
x_{*}=\min \{y \in[a, b] \mid y \geq Q y\} \quad \text { and } \quad x^{*}=\max \{y \in[a, b] \mid y \leq Q y\} .
$$

In the following section, we combine Theorems 2.1, and 2.2 to obtain a general hybrid fixed point theorem for the mappings on ordered Banach spaces.

\section{Hybrid fixed point theory}

Our main hybrid fixed point theorem of this paper is 
Theorem 3.1. Let $[a, b]$ be a norm-bounded order interval in the ordered $B a$ nach space $X$ and let $T:[a, b] \times[a, b] \rightarrow[a, b]$ be a mapping satisfying the following conditions.

(a) The mapping $x \mapsto T(x, y)$ is continuous uniformly for $y \in[a, b]$,

(b) The mapping $x \mapsto T(x, y)$ is countably condensing and monotone increasing for each $y \in[a, b]$,

(c) $y \mapsto T(x, y)$ is monotone increasing for each $x \in[a, b]$, and

(d) every sequence $\left\{T\left(x, y_{n}\right)\right\} \subseteq T([a, b] \times[a, b])$ converges for each $x \in$ $[a, b]$, whenever $\left\{y_{n}\right\}$ is a monotone sequence in $[a, b]$.

Then the operator equation $x=T(x, x)$ has the least and the greatest solution in $[a, b]$.

Proof. Define the operator $Q:[a, b] \rightarrow[a, b]$ by

$$
Q y=z,
$$

where $z$ is the greatest solution of the operator equation $T(z, y)=z$. Let $y \in$ $[a, b]$ be fixed and define the operator $T_{y}(x):[a, b] \rightarrow[a, b]$ by $T_{y}(x)=T(x, y)$. Then $T_{y}$ is a countably condensing, continuous and monotone increasing operator which maps a closed convex and bounded subset $[a, b]$ of the Banach space $X$ into itself. Therefore, an application of Theorem 2.1 yields that $T_{y}$ has the least and the greatest fixed point in $[a, b]$ and consequently the mapping $Q$ is well defined for each $y \in[a, b]$. Next we show that $Q$ satisfies all the requirement of Theorem 2.2 on $[a, b]$.

First, we show that $Q$ is a nondecreasing mapping on $[a, b]$. Let $y_{1}, y_{2} \in[a, b]$ be such that $y_{1} \leq y_{2}$. Then there are unique elements $z_{1}, z_{2} \in[a, b]$ such that

$$
Q y_{1}=z_{1}=T\left(z_{1}, y_{1}\right)=T_{y_{1}}\left(z_{1}\right)
$$

and

$$
Q y_{2}=z_{2}=T\left(z_{2}, y_{2}\right)=T_{y_{2}}\left(z_{2}\right)
$$

From the monotonicity of $T(x, y)$ in $y$, it follows that

$$
T_{y_{1}}(x)=T\left(x, y_{1}\right) \leq T\left(x, y_{2}\right)=T_{y_{2}}(x)
$$

for all $x \in[a, b]$. Hence for any $x \in[a, b]$

$$
T_{y_{1}}^{n}(x) \leq T_{y_{2}}^{n}(x)
$$

for all $n \in \mathbb{N}$. In particular,

$$
T_{y_{1}}^{n}(b) \leq T_{y_{2}}^{n}(b)
$$

for all $n \in \mathbb{N}$. By Theorem 2.1,

$$
z_{1}=\lim _{n \rightarrow \infty} T_{y_{1}}^{n}(b) \leq \lim _{n \rightarrow \infty} T_{y_{2}}^{n}(b)=z_{2} .
$$

This shows that $Q$ defines a nondecreasing operator $Q:[a, b] \rightarrow[a, b]$ (see also Dhage $[1,2]$ and the references therein).

Next, let $\left\{y_{n}\right\}$ be a monotone sequence in $[a, b]$. We will show that the sequence $\left\{Q y_{n}\right\}$ converges. By virtue of $Q$, there is a monotone increasing 
sequence $\left\{z_{n}\right\}$ in $[a, b]$ such that $z_{n}=T\left(z_{n}, y_{n}\right), n \in \mathbb{N}$. Let $S=\left\{z_{n}\right\}$. Then $S$ is a bounded and countable subset of $[a, b]$ such that $S \subseteq \bigcup_{n \in \mathbb{N}} T\left(S, y_{n}\right)$. Since the map $x \mapsto T(x, y)$ is countably condensing for each $y \in[a, b]$, one has

$$
\alpha(S) \leq \alpha\left(\bigcup_{n \in \mathbb{N}} T\left(S, y_{n}\right)\right)=\sup \left\{\alpha\left(T\left(S, y_{n}\right)\right): n \in \mathbb{N}\right\}<\alpha(S) .
$$

If $\alpha(S) \neq 0$, then we get a contradiction. As a result $\alpha(S)=0$ and that $\bar{S}$ is compact. Hence the sequence $\left\{z_{n}\right\}$ converges to a point, say $z$ in $[a, b]$. Now, by hypothesis (c), the sequence $\left\{T\left(z, y_{n}\right)\right\}$ converges, say to the point $T(z, y)$ for some $y \in[a, b]$. Then, we have

$$
\left\|T\left(z_{n}, y_{n}\right)-T(z, y)\right\| \leq\left\|T\left(z_{n}, y_{n}\right)-T\left(z, y_{n}\right)\right\|+\left\|T\left(z, y_{n}\right)-T(z, y)\right\| .
$$

Passing the limit to $n \rightarrow \infty$ in the above inequality,

$$
\lim _{n \rightarrow \infty}\left\|T\left(z_{n}, y_{n}\right)-T(z, y)\right\|=0 .
$$

As a result, the sequence $\left\{Q y_{n}\right\} \subseteq Q([a, b])$ converges, whenever $\left\{y_{n}\right\}$ is a monotone sequence in $[a, b]$.

Thus, $Q$ satisfies all the conditions of Theorem 2.2 on $[a, b]$ and hence an application it yields that $Q$ has the least and the greatest fixed point. The greatest fixed point of $Q$ is the greatest solution of the operator equation $x=$ $T(x, x)$ in $[a, b]$. Similarly, define the operator $P:[a, b] \rightarrow[a, b]$ by

$$
P y=z,
$$

where $z$ is the least solution of the operator equation $T(z, y)=z$. Clearly the operator $P$ is well defined. It can be shown by using the similar arguments that $P$ has the least fixed point in $[a, b]$ which is also the least solution of the operator equation $T(x, x)=x$. As a result, the operator equation $T(x, x)=x$ has the least and the greatest solution in $[a, b]$. This completes the proof.

As a consequence of Theorem 3.1 we obtain

Corollary 3.1. Let $[a, b]$ be an order interval in the ordered Banach space $X$ and let $T:[a, b] \times[a, b] \rightarrow[a, b]$ be a mapping satisfying

(a) $x \mapsto T(x, y)$ is continuous uniformly for $y \in[a, b]$,

(b) $x \mapsto T(x, y)$ is countably condensing and monotone increasing for all $y \in[a, b]$, and

(c) $y \mapsto T(x, y)$ is monotone increasing for each $x \in[a, b]$.

Then the operator equation $x=T(x, x)$ has the least and the greatest solution if any one of the following conditions is satisfied.

(i) $[a, b]$ is norm-bounded and $T$ is compact multi-map.

(ii) The cone $K$ in $X$ is normal and $y \mapsto T(x, y)$ is a compact for each $x \in[a, b]$.

(iii) The cone $K$ is regular. 
The origin of hybrid fixed point theorems involving the sum of the two operators in a Banach space lies in the works of the Russian mathematician Krasnoselskii [10]. In this case one operator happens to be a contraction and another one happens to be a completely continuous on the domains of their definitions. Since every contraction is continuous, both the operators in such theorems are continuous. Below we prove a hybrid fixed point theorem involving the sum of three operators in Banach spaces and relax the continuity condition of one of the mappings in such hybrid fixed point theorems, instead we assume the monotonicity and prove a fixed point theorem on ordered Banach spaces.

To prove the main result in this direction, we need the following useful lemma.

Lemma 3.1. Let $A, B: X \rightarrow X$ be two mappings such that

(a) $A$ is a nonlinear $\mathcal{D}$-contraction and

(b) $B$ is completely continuous.

Then the map $T: X \rightarrow X$ defined by $T x=A x+B x$ is a continuous and condensing on $X$.

Proof. The proof appears in Dhage [1].

Theorem 3.2. Let $[a, b]$ be an order interval in an ordered Banach space $X$. Let $A, B, C:[a, b] \rightarrow X$ be three monotone increasing operators satisfying

(a) $A$ is a nonlinear $\mathcal{D}$-contraction,

(b) $B$ is completely continuous, and

(c) every sequence $\left\{C z_{n}\right\} \subseteq C([a, b])$ converges whenever $\left\{z_{n}\right\}$ is a monotone sequence in $[a, b]$, and

(d) $A x+B y+C z \in[a, b]$ for all $x, y, z \in[a, b]$.

Further, if the cone $K$ in $X$ is normal, then the operator equation $A x+B x+$ $C x=x$ has the least and the greatest solution in $[a, b]$.

Proof. Define an operator $T$ on $[a, b] \times[a, b]$ by $T(x, y)=A x+B x+C y$. From hypothesis $(d)$ it follows that $T$ defines a mapping $T:[a, b] \times[a, b] \rightarrow[a, b]$. From Lemma 3.1, it follows that the map $x \mapsto T(x, y)$ is condensing, continuous and monotone increasing on $[a, b]$ uniformly for $y \in[a, b]$. Now the desired conclusion follows by an application of Theorem 3.1.

Corollary 3.2. (Dhage [2]) Let $[a, b]$ be an order interval in the ordered Banach space $X$. Let $A, B, C:[a, b] \rightarrow X$ be three nondecreasing operators satisfying

(a) $A$ is a contraction,

(b) $B$ is completely continuous and

(c) $C$ is compact, and

(d) $A x+B y+C z \in[a, b]$ for all $x, y, z \in[a, b]$.

Further, if the cone $K$ in $X$ is normal, then the operator equation $A x+B x+$ $C x=x$ has the least and the greatest solution in $[a, b]$. 
Remark 3.1. Note that hypothesis $(d)$ holds in Theorem 3.1 and Corollary 3.2, if the operators $A, B, C$ are monotone increasing and there exist elements $a$ and $b$ in $X$ such that $a \leq A a+B a+C a$ and $A b+B b+C b \leq b$ satisfying $a \leq b$.

Remark 3.2. Notice that Corollary 3.2 is an improvement upon a fixed point theorem proved in Dhage [3] concerning the solutions of operator equations involving the three operators in Banach spaces.

Theorem 3.2 contains two hybrid fixed point theorems useful in applications to nonlinear differential and integral equations, because of their special importance we state them separately.

Corollary 3.3. Let $[a, b]$ be an order interval in the ordered Banach space $X$. Let $A, B:[a, b] \rightarrow X$ be two monotone increasing operators satisfying

(a) $A$ is completely continuous, and

(b) every sequence $\left\{B y_{n}\right\} \subseteq B([a, b])$ converges, whenever $\left\{y_{n}\right\}$ is a monotone sequence in $[a, b]$, and

(c) $A x+B y \in[a, b]$ for all $x, y \in[a, b]$.

Further, if the cone $K$ in $X$ is normal, then the operator equation $A x+B x=x$ has the least and the greatest solution in $[a, b]$.

Corollary 3.4. Let $[a, b]$ be an order interval in the ordered Banach space $X$. Let $A, B:[a, b] \rightarrow X$ be two monotone increasing operators satisfying

(a) $A$ is a nonlinear $\mathcal{D}$-contraction, and

(b) every sequence $\left\{B y_{n}\right\} \subseteq B([a, b])$ converges, whenever $\left\{y_{n}\right\}$ is a monotone sequence in $[a, b]$, and

(c) $A x+B y \in[a, b]$ for all $x, y \in[a, b]$.

Further, if the cone $K$ in $X$ is normal, then the operator equation $A x+B x=x$ has the least and the greatest solution in $[a, b]$.

Remark 3.3. Note that hypothesis (c) holds in Corollary 3.3 and Corollary 3.4, if the operators $A, B$ are monotone increasing and there exist elements $a$ and $b$ in $X$ such that $a \leq A a+B a$ and $A b+B b \leq b$ satisfying $a \leq b$.

\section{Existence theory}

Let $B(I, \mathbb{R})$ denote the space of bounded real-valued functions on $I$. Let $C(J, \mathbb{R})$, denote the space of all continuous real-valued functions on $J$. Define a norm $\|\cdot\|$ by

$$
\|x\|=\sup _{t \in J}|x(t)| \text {. }
$$

Clearly $C(J, \mathbb{R})$ becomes a Banach space with respect to the above norm. By $L^{1}(I, \mathbb{R})$ we denote the set of Lebesgue integrable functions on $I$ and the norm $\|\cdot\|_{L^{1}}$ in $L^{1}(I, \mathbb{R})$ is defined by

$$
\|x\|_{L^{1}}=\int_{0}^{T}|x(t)| d s .
$$


Define the order relation " $\leq$ " by the cone $K$ in $C(J, \mathbb{R})$, given by

$$
K=\{x \in C(J, \mathbb{R}) \mid x(t) \geq 0 \text { for all } t \in J\} .
$$

Clearly, the cone $K$ is normal in $C(J, \mathbb{R})$. Note that the order relation " $\leq$ " in $C(J, \mathbb{R})$ also induces the order relation in the space $\mathcal{C}=C\left(I_{0}, \mathbb{R}\right)$ which we also denote by " $\leq$ " itself when there is no confusion.

We need the following definitions in the sequel.

Definition 4.1. A mapping $\beta: I \times \mathcal{C} \rightarrow \mathbb{R}$ is called $\mathcal{D}$-Lipschitz if there exists a continuous and nondecreasing function $\psi$ such that

$$
|\beta(t, x)-\beta(t, y)| \leq \psi\left(\|x-y\|_{\mathcal{C}}\right)
$$

for all $(t, x),(t, y) \in I \times \mathcal{C}$. The function $\psi$ is called a $\mathcal{D}$-function of $\beta$ on $I \times \mathcal{C}$.

Definition 4.2. A mapping $\beta: I \times \mathcal{C} \rightarrow \mathbb{R}$ is said to be Carathéodory if

(i) $t \mapsto \beta(t, x)$ is measurable for each $x \in \mathcal{C}$, and

(ii) $x \mapsto \beta(t, x)$ is continuous almost everywhere for $t \in I$.

Furthermore, a Carathéodory function $\beta(t, x)$ is called $L^{1}$-Carathéodory if

(iii) for each real number $r>0$ there exists a function $h_{r} \in L^{1}(I, \mathbb{R})$ such that

$$
|\beta(t, x)| \leq h_{r}(t), \quad \text { a.e. } t \in I
$$

for all $x \in \mathcal{C}$ with $\|x\|_{\mathcal{C}} \leq r$.

Definition 4.3. A mapping $\beta: I \times \mathcal{C} \rightarrow \mathbb{R}$ is said to be Chandrabhan if

(i) $t \mapsto \beta\left(t, x_{t}\right)$ is Lebesgue integrable for each $x \in C(J, \mathbb{R})$, and

(ii) $x \mapsto \beta(t, x)$ is nondecreasing almost everywhere for $t \in I$.

Furthermore, a Chandrabhan function $\beta(t, x)$ is called $L^{1}$-Chandrabhan if

(iii) for each real number $r>0$ there exists a function $q_{r} \in L^{1}(I, \mathbb{R})$ such that

$$
|\beta(t, x)| \leq q_{r}(t), \quad \text { a.e. } t \in I
$$

for all $x \in \mathcal{C}$ with $\|x\|_{\mathcal{C}} \leq r$.

\subsection{Existence results for first order NFDE}

Definition 4.4. A function $x \in C(J, \mathbb{R}) \cap A C(I, \mathbb{R})$ is called a solution of the NFDE (1.1) on $J$ if

(i) the function $t \mapsto\left[x(t)-f\left(t, x_{t}\right)\right]$ is absolutely continuous on $I$, and

(ii) $x$ satisfies the equations in (1.1),

where $A C(I, \mathbb{R})$ is the space of absolutely continuous real-valued functions on $I$.

We use the following definitions in the sequel. 
Definition 4.5. A function $a \in C(J, \mathbb{R}) \cap A C(I, \mathbb{R})$ is called a lower solution of the NFDE (1.1) on $J$ if the map $t \mapsto\left[a(t)-f\left(t, a_{t}\right)\right]$ is absolutely continuous on $I$, and

$$
\left.\begin{array}{rl}
\frac{d}{d t}\left[a(t)-f\left(t, a_{t}\right)\right] & \leq g\left(t, a_{t}\right)+k\left(t, a_{t}\right) \text { a.e. } t \in I \\
a_{0} & \leq \phi .
\end{array}\right\}
$$

Again, a function $b \in C(J, \mathbb{R}) \cap A C(I, \mathbb{R})$ is called an upper solution of the NFDE (1.1) on $J$ if the function $t \mapsto\left[b(t)-f\left(t, b_{t}\right)\right]$ is absolutely continuous on $I$, and

$$
\left.\begin{array}{rl}
\frac{d}{d t}\left[b(t)-f\left(t, b_{t}\right)\right] & \geq g\left(t, b_{t}\right)+k\left(t, b_{t}\right) \text { a.e. } t \in I \\
b_{0} & \geq \phi .
\end{array}\right\}
$$

Finally, a function $x \in C(J, \mathbb{R}) \cap A C(I, \mathbb{R})$ is a solution of the NFDE (1.1) on $J$ if it is a lower as well as a upper solution of the NFDE (1.1) on $J$.

Definition 4.6. A solution $x_{M}$ of the NFDE (1.1) is said to be maximal if for any other solution $x$ to NFDE (1.1) one has $x(t) \leq x_{M}(t)$ for all $t \in J$. Again, a solution $x_{m}$ of the NFDE (1.1) is said to be minimal if $x_{m}(t) \leq x(t)$ for all $t \in J$, where $x$ is any solution for the NFDE (1.1) on $J$.

We need the following hypotheses in the sequel.

$\left(f_{0}\right) f(0, x)=0$ for all $x \in \mathcal{C}$.

$\left(f_{1}\right) f$ is continuous and $\mathcal{D}$-Lipschitz on $I \times \mathcal{C}$ with the $\mathcal{D}$-function $\psi$.

$\left(f_{2}\right)$ The function $f(t, x)$ is nondecreasing in $x$ almost everywhere for $t \in I$.

$\left(g_{1}\right) g$ is $L^{1}$-Carathéodory.

$\left(g_{2}\right)$ The function $g(t, x)$ is nondecreasing in $x$ almost everywhere for $t \in I$.

$\left(k_{1}\right) k$ is $L^{1}$-Chandrabhan.

$\left(k_{2}\right)$ NFDE (1.1) has a lower solution $a$ and an upper solution $b$ with $a \leq b$.

Theorem 4.1. Assume that the hypotheses $\left(f_{0}\right)-\left(f_{2}\right),\left(g_{1}\right)-\left(g_{2}\right)$ and $\left(k_{1}\right)-\left(k_{2}\right)$ hold. If $\psi(r)<r$ for $r>0$, then the NFDE (1.1) has a minimal and a maximal solution in $[a, b]$ defined on $J$.

Proof. NFDE (1.1) is equivalent to the integral equation

$$
x(t)= \begin{cases}\phi(0)-f(0, \phi)+f\left(t, x_{t}\right)+\int_{0}^{t} g\left(s, x_{s}\right) d s+\int_{0}^{t} k\left(s, x_{s}\right) d s, & \text { if } t \in I \\ \phi(t), & \text { if } t \in I_{0} .\end{cases}
$$

Set $X=C(J, \mathbb{R})$ and consider the order interval $[a, b]$ in $X$ which is well defined in view of hypothesis $\left(k_{2}\right)$. Define three operators $A, B, C:[a, b] \rightarrow X$ by

$$
A x(t)= \begin{cases}-f(0, \phi)+f\left(t, x_{t}\right), & \text { if } t \in I, \\ 0, & \text { if } t \in I_{0},\end{cases}
$$




$$
B x(t)= \begin{cases}\phi(0)+\int_{0}^{t} g\left(s, x_{s}\right) d s, & \text { if } t \in I, \\ \phi(t), & \text { if } t \in I_{0},\end{cases}
$$

and

$$
C x(t)= \begin{cases}\int_{0}^{t} k\left(s, x_{s}\right) d s, & \text { if } t \in I, \\ 0, & \text { if } t \in I_{0} .\end{cases}
$$

Clearly the operators $A, B$ and $C$ are well defined on $[a, b]$ in view of hypotheses $\left(f_{1}\right),\left(g_{1}\right)$ and $\left(k_{1}\right)$. Then the integral equation $(4.2)$ is equivalent to the operator equation

$$
A x(t)+B x(t)+C x(t)=x(t), \quad t \in J .
$$

We shall show that $A, B$ and $C$ satisfy all the conditions of Corollary 3.2 on $[a, b]$.

Step I : Firstly, we show that $A$ is a contraction operator on $[a, b]$. Let $x, y \in[a, b]$. Then by hypothesis $\left(f_{1}\right)$,

$$
\|A x-A y\| \leq \sup _{t \in J}\left|f\left(t, x_{t}\right)-f\left(t, y_{t}\right)\right| \leq \psi\left(\left\|x_{t}-y_{t}\right\|_{\mathcal{C}}\right) \leq \psi(\|x-y\|)
$$

where, $\psi(r)<r$ for $r>0$. This shows that $A$ is a nonlinear $\mathcal{D}$-contraction on $[a, b]$. Next we show that $A$ is monotone increasing on $[a, b]$. Let $x, y \in[a, b]$ be such that $x \leq y$. Then, by $\left(f_{2}\right)$,

$$
\begin{aligned}
A x(t) & = \begin{cases}-f(0, \phi)+f\left(t, x_{t}\right), & \text { if } t \in I, \\
0, & \text { if } t \in I_{0},\end{cases} \\
& \leq \begin{cases}-f(0, \phi)+f\left(t, y_{t}\right), & \text { if } t \in I, \\
0, & \text { if } t \in I_{0},\end{cases} \\
& =A y(t)
\end{aligned}
$$

for all $t \in J$. Hence, $A x \leq A y$, and so, the operator $A$ is monotone increasing on $[a, b]$.

Step II : Secondly, we show that the operator $B$ is completely continuous on $[a, b]$. It can be shown as in Guenther et al. [6] that $B$ is a continuous operator on $[a, b]$. Now, we show, $B$ maps bounded sets into bounded sets in $X$. If $S$ is a bounded set in $X$, then there exists $r>0$ such that $\|x\| \leq r$ for all $x \in S$. Now for each $u \in B(S)$, one has

$$
u(t)= \begin{cases}\phi(0)+\int_{0}^{t} g\left(s, x_{s}\right) d s, & \text { if } t \in I, \\ \phi(t), & \text { if } t \in I_{0}\end{cases}
$$


for some $x \in S$. Then, for each $t \in J$,

$$
\begin{aligned}
|u(t)| & \leq\|\phi\|_{\mathcal{C}}+\int_{0}^{t}\left|g\left(s, x_{s}\right)\right| d s \\
& \leq\|\phi\|_{\mathcal{C}}+\int_{0}^{t} h_{r}(s) d s \\
& \leq\|\phi\|_{\mathcal{C}}+\left\|h_{r}\right\|_{L^{1}} .
\end{aligned}
$$

This further implies that

$$
\|u\| \leq\|\phi\|_{\mathcal{C}}+\left\|h_{r}\right\|_{L^{1}}
$$

for all $u \in B(S)$. Hence, $B(S)$ is bounded.

Next we show that $B$ maps bounded sets into equicontinuous sets. Let $S$ be, as above. Then, we have

$$
u(t)= \begin{cases}\phi(0)+\int_{0}^{t} g\left(s, x_{s}\right) d s, & \text { if } t \in I, \\ \phi(t), & \text { if } t \in I_{0}\end{cases}
$$

for some $x \in S$. Then for any $t_{1}, t_{2} \in I$ with $t_{1} \leq t_{2}$, we have

$$
\begin{aligned}
\left|u\left(t_{1}\right)-u\left(t_{2}\right)\right| & \leq\left|\int_{0}^{t_{1}} g\left(s, x_{s}\right) d s-\int_{0}^{t_{2}} g\left(s, x_{s}\right) d s\right| \\
& =\int_{t_{1}}^{t_{2}}\left|g\left(s, x_{s}\right)\right| d s \\
& \leq \int_{t_{1}}^{t_{2}} h_{r}(s) d s .
\end{aligned}
$$

If $t_{1}, t_{2} \in I_{0}$, then $\left|u\left(t_{1}\right)-u\left(t_{2}\right)\right|=\left|\phi\left(t_{1}\right)-\phi\left(t_{2}\right)\right|$. For the case, where $t_{1} \leq 0 \leq$ $t_{2}$, we have that

$$
\begin{aligned}
\left|u\left(t_{1}\right)-u\left(t_{2}\right)\right| & \leq\left|\phi\left(t_{1}\right)-\phi(0)-\int_{0}^{t_{2}} g\left(s, x_{s}\right) d s\right| \\
& \leq\left|\phi\left(t_{1}\right)-\phi(0)\right|+\int_{0}^{t_{2}}\left|g\left(s, x_{s}\right)\right| d s \\
& \leq\left|\phi\left(t_{1}\right)-\phi(0)\right|+\int_{0}^{t_{2}} h_{r}(s) d s .
\end{aligned}
$$

Hence, in all cases, we have

$$
\left|u\left(t_{1}\right)-u\left(t_{2}\right)\right| \rightarrow 0 \text { as } t_{1} \rightarrow t_{2}
$$

As a result $B(S)$ is an equicontinuous set in $X$. Now an application of ArzeláAscoli theorem yields that the operator $B$ is totally bounded on $X$. Again, it can be proved as in the case of operator $A$, that the operator $B$ is also monotone increasing on $[a, b]$.

Step III : Finally, we show that the operator $C$ satisfies the hypothesis (c) of Theorem 3.2 on $[a, b]$. Let $\left\{z_{n}\right\}$ be a monotone sequence in $[a, b]$. We show 
that the sequence $\left\{C z_{n}\right\}$ converges in $X$. From the monotonicity of $k(t, x)$ in $x$, it follows that the operator $C$ and consequently the sequence $\left\{C z_{n}\right\}$ is also monotone in $X$. To finish, it is enough to show that $\left\{C z_{n}\right\}$ is uniformly bounded and equicontinuous sequence. Since the cone $K$ in $X$ is normal, there exists a constant $r>0$ such that $\left\|z_{n}\right\| \leq r$ for all $n \in \mathbb{N}$. By the definition of $C$, one has

$$
C z_{n}(t)= \begin{cases}\int_{0}^{t} k\left(s, z_{n}(s+\theta)\right) d s, & \text { if } t \in I, \\ 0, & \text { if } t \in I_{0} .\end{cases}
$$

Since $\left(H_{3}\right)$ holds, we have

$$
\begin{aligned}
\left|C z_{n}(t)\right| & \leq \int_{0}^{t}\left|k\left(s, z_{n}(s+\theta)\right)\right| d s \\
& \leq \int_{0}^{t} q_{r}(s) d s \\
& \leq\left\|q_{r}\right\|_{L^{1}}
\end{aligned}
$$

for all $t \in J$. This implies that

$$
\left\|C z_{n}\right\| \leq\left\|q_{r}\right\|_{L^{1}}
$$

for all $n \in \mathbb{N}$. Hence, $\left\{C z_{n}\right\}$ is uniformly bounded.

Next we show that $\left\{C z_{n}\right\}$ is equicontinuous sequence in $X$. Now, for any $t_{1}, t_{2} \in I$ with $t_{1} \leq t_{2}$, we have

$$
\begin{aligned}
\left|C z_{n}\left(t_{1}\right)-C z_{n}\left(t_{2}\right)\right| & \leq\left|\int_{0}^{t_{1}} k\left(s, z_{n}(s+\theta)\right) d s-\int_{0}^{t_{2}} k\left(s, z_{n}(s+\theta)\right) d s\right| \\
& =\int_{t_{1}}^{t_{2}}\left|k\left(s, z_{n}(s+\theta)\right)\right| d s \\
& \leq \int_{t_{1}}^{t_{2}} q_{r}(s) d s .
\end{aligned}
$$

If $t_{1}, t_{2} \in I_{0}$, then $\left|C z_{n}\left(t_{1}\right)-C z_{n}\left(t_{2}\right)\right|=0$. For the case, where $t_{1} \leq 0 \leq t_{2}$ we have that

$$
\left|C z_{n}\left(t_{1}\right)-C z_{n}\left(t_{2}\right)\right| \leq\left|\int_{0}^{t_{2}} k\left(s, z_{n}(s+\theta)\right) d s\right| \leq\left|p\left(t_{2}\right)-p(0)\right|
$$

where $p(t)=\int_{0}^{t} q_{r}(s) d s$.

Hence, in all cases, we have

$$
\left|C z_{n}\left(t_{1}\right)-C z_{n}\left(t_{2}\right)\right| \rightarrow 0 \text { as } t_{1} \rightarrow t_{2}
$$

As a result $\left\{C z_{n}\right\}$ is an equicontinuous sequence in $X$. Now an application of Arzelá-Ascoli theorem yields that the sequence $\left\{C z_{n}\right\}$ converges in $X$.

Thus the operators $A, B$ and $C$ satisfy all the conditions of Theorem 3.2 and hence the operator equation $A x+B x+C x=x$ and consequently the 
NFDE (1.1)has a minimal and a maximal solution in $[a, b]$ defined on $J$. This completes the proof.

\subsection{Existence results for second order NFDE}

We use the following definitions in the sequel.

Definition 4.7. A function $x \in C(J, \mathbb{R}) \cap A C^{1}(I, \mathbb{R})$ is called a solution of the NFDE (1.1) on $J$ if

(i) the function $t \mapsto\left[x^{\prime}(t)-f\left(t, x_{t}\right)\right]$ is absolutely continuous on $I$, and

(ii) $x$ satisfies the equations in (1.1),

where $A C^{1}(I, \mathbb{R})$ is the space of continuous real-valued functions whose first derivative exists and is absolutely continuous on $I$.

Definition 4.8. A function $a \in C(J, \mathbb{R}) \cap A C^{1}(I, \mathbb{R})$ is called a lower solution of the NFDE (1.2) on $J$ if the map $t \mapsto\left[a^{\prime}(t)-f\left(t, a_{t}\right)\right]$ is absolutely continuous on $I$, and

$$
\left.\begin{array}{c}
\frac{d}{d t}\left[a^{\prime}(t)-f\left(t, a_{t}\right)\right] \leq g\left(t, a_{t}\right)+k\left(t, a_{t}\right) \text { a.e. } t \in I \\
a_{0} \leq \phi, \quad a^{\prime}(0) \leq \eta
\end{array}\right\}
$$

Again, a function $b \in C(J, \mathbb{R}) \cap A C^{1}(J, \mathbb{R})$ is called an upper solution of the NFDE (1.2) on $J$ if the map $t \mapsto\left[b^{\prime}(t)-f\left(t, b_{t}\right)\right]$ is absolutely continuous on $I$, and

$$
\left.\begin{array}{c}
\frac{d}{d t}\left[b^{\prime}(t)-f\left(t, b_{t}\right)\right] \geq g\left(t, b_{t}\right)+k\left(t, b_{t}\right) \text { a.e. } t \in I \\
b_{0} \geq \phi, \quad b^{\prime}(0) \geq \eta
\end{array}\right\}
$$

Finally, a function $x \in C(J, \mathbb{R}) \cap A C^{1}(I, \mathbb{R})$ is a solution of the NFDE (1.2) on $J$ if it is a lower as well as a upper solution of the NFDE (1.2) on $J$.

Definition 4.9. A solution $x_{M}$ of the NFDE (1.2) is said to be maximal if for any other solution $x$ to NFDE (1.2) one has $x(t) \leq x_{M}(t)$ for all $t \in J$. Again, a solution $x_{m}$ of the NFDE (1.2) is said to be minimal if $x_{m}(t) \leq x(t)$ for all $t \in J$, where $x$ is any solution of the NFDE (1.2) on $J$.

We need the following hypothesis in the sequel.

$\left(k_{3}\right)$ NFDE (1.2) has a lower solution $a$ and an upper solution $b$ with $a \leq b$.

Theorem 4.2. Assume that the hypotheses $\left(f_{1}\right)-\left(f_{2}\right),\left(g_{1}\right)-\left(g_{2}\right)$ and $\left(k_{1}\right),\left(k_{3}\right)$ hold. Furthermore, if $T \psi(r)<r$ for $r>0$, then the NFDE (1.2) has a minimal and a maximal solution in $[a, b]$ defined on $J$. 
Proof. NFDE (1.2) is equivalent to the integral equation

$$
x(t)=\left\{\begin{array}{rlrl}
\phi(0)+[\eta-f(0, \phi)] t+\int_{0}^{t} f\left(s, x_{s}\right) d s & \\
& +\int_{0}^{t}(t-s) g\left(s, x_{s}\right) d s+\int_{0}^{t}(t-s) k\left(s, x_{s}\right) d s, & & \text { if } t \in I \\
\phi(t), & & \text { if } t \in I_{0} .
\end{array}\right.
$$

Set $X=C(J, \mathbb{R})$ and consider the order interval $[a, b]$ in $X$ which is well defined in view of hypothesis $\left(k_{3}\right)$. Define three operators $A, B, C:[a, b] \rightarrow X$ by

$$
\begin{gathered}
A x(t)= \begin{cases}{[\eta-f(0, \phi)] t+\int_{0}^{t} f\left(s, x_{s}\right) d s,} & \text { if } t \in I, \\
0, & \text { if } t \in I_{0},\end{cases} \\
B x(t)= \begin{cases}\phi(0)+\int_{0}^{t}(t-s) g\left(s, x_{s}\right) d s, & \text { if } t \in I, \\
\phi(t), & \text { if } t \in I_{0},\end{cases}
\end{gathered}
$$

and

$$
C x(t)= \begin{cases}\int_{0}^{t}(t-s) k\left(s, x_{s}\right) d s, & \text { if } t \in I, \\ 0, & \text { if } t \in I_{0} .\end{cases}
$$

Clearly the operators $A, B$ and $C$ are well defined on $[a, b]$ in view of hypotheses $\left(f_{1}\right),\left(g_{1}\right)$ and $\left(k_{1}\right)$. Then, the integral equation (4.7) is equivalent to the operator equation

$$
A x(t)+B x(t)+C x(t)=x(t), \quad t \in J .
$$

Now, it can shown as in the proof of Theorem 4.1, that the operators $A, B$ and $C$ satisfy all the conditions of Theorem 3.2 on $[a, b]$. Hence NDFE (1.2) has a minimal and a maximal solution in $[a, b]$ defined on $J$.

The existence results for extremal solutions of Carathéodory NFDEs (1.3) and (1.4) can be stated as follows. For this, we need the following hypotheses in the sequel.

$\left(k_{4}\right)$ NFDE (1.3) has a lower solution $a$ and an upper solution $b$ with $a \leq b$.

$\left(k_{5}\right)$ NFDE (1.4) has a lower solution $a$ and an upper solution $b$ with $a \leq b$.

Theorem 4.3. Assume that the hypotheses $\left(f_{1}\right)-\left(f_{2}\right),\left(g_{1}\right)-\left(g_{2}\right)$ and $\left(k_{4}\right)$ hold. Furthermore, if $\psi(r)<r$ for $r>0$, then the NFDE (1.3) has a minimal and a maximal solution in $[a, b]$ defined on $J$.

Theorem 4.4. Assume that the hypotheses $\left(f_{1}\right)-\left(f_{2}\right),\left(g_{1}\right)-\left(g_{2}\right)$ and $\left(k_{5}\right)$ hold. Furthermore, if $T \psi(r)<r$ for $r>0$, then the NFDE (1.4) has a minimal and a maximal solution in $[a, b]$ defined on $J$. 
Similarly, the existence results for the extremal solutions of discontinuous NFDE (1.3) and (1.4) can be stated as follows.

Theorem 4.5. Assume that the hypotheses $\left(f_{1}\right)-\left(f_{2}\right),\left(k_{1}\right)$ and $\left(k_{3}\right)$ hold with the function $k$ replaced by $g$. Furthermore, if $\psi(r)<r$ for $r>0$, then the NFDE (1.3) has a minimal and a maximal solution in $[a, b]$ defined on $J$.

Theorem 4.6. Assume that the hypotheses $\left(f_{1}\right)-\left(f_{2}\right),\left(k_{1}\right)$ and $\left(k_{4}\right)$ hold with the function $k$ replaced by $g$. If $T \psi(r)<r$ for $r>0$, then the NFDE (1.4) has a minimal and a maximal solution in $[a, b]$ defined on $J$.

\section{References}

[1] B. C. Dhage, Existence theory for nonlinear functional perturbed differential equations, Nonlinear Anal. Forum 10 (2005), no. 1, 69-80.

[2] Existence of extremal solutions for discontinuous functional integral equations, Appl. Math. Lett. 19 (2006), no. 9, 881-886.

[3] — A general multi-valued hybrid fixed point theorem and perturbed differential inclusions, Nonlinear Anal. 64 (2006), no. 12, 2747-2772.

[4] K. Deimling, Nonlinear functional analysis, Springer-Verlag, Berlin, 1985.

[5] A. Granas and J. Dugundji, Fixed point theory, Springer Monographs in Mathematics. Springer-Verlag, New York, 2003.

[6] A. Granas, R. B. Guenther, and J. W. Lee, Some general existence principles in the Caratheodory theory of nonlinear differential systems, J. Math. Pures Appl. (9) 70 (1991), no. 2, 153-196.

[7] J. K. Hale, Theory of Functional Differential Equations, Springer, New York, 1977.

[8] S. Heikkilä and V. Lakshmikantham, Monotone iterative techniques for discontinuous nonlinear differential equations, Monographs and Textbooks in Pure and Applied Mathematics, 181. Marcel Dekker, Inc., New York, 1994.

[9] J. Henderson, Boundary value problems for functional-differential equations, World Scientific Publishing Co., Inc., River Edge, NJ, 1995.

[10] M. A. Krasnoselskii, Topological Methods in the Theory of Nonlinear Integral Equations, Pergamon Press, 1964.

[11] S. K. Ntouyas, Initial and boundary value problems for functional-differential equations via the topological transversality method: a survey, Bull. Greek Math. Soc. 40 (1998), $3-41$.

[12] S. K. Ntouyas, Y. G. Sficas, and P. Ch. Tsamatos, Existence results for initial value problems for neutral functional-differential equations, J. Differential Equations 114 (1994), no. $2,527-537$.

[13] Y. G. Sficas and S. K. Ntouyas, A two-point boundary value problem for neutral functional-differential equations, Proc. Roy. Soc. Edinburgh Sect. A 94 (1983), no. 3-4, 331-338.

[14] S. K. Ntouyas and P. Ch. Tsamatos, Global existence for functional integro-differential equations of delay and neutral type, Appl. Anal. 54 (1994), no. 3-4, 251-262.

[15] E. Zeidler, Nonlinear Functional Analysis: Part I, Springer Verlag, New York, 1985.

Kasubai, Gurukul Colony

AHMEDPUR-413 515

Dist: LATUR, MAHARASHTRA, INDIA

E-mail address: bcd20012001@yahoo.co.in 